\title{
Highly Sensitive and Selective Eco-Toxic 4-Nitrophenol Chemical Sensor Based on Ag-Doped ZnO Nanoflowers Decorated with Nanosheets
}

\author{
Ahmad Umar ${ }^{1,2, *(\mathbb{D})}$, M. Shaheer Akhtar ${ }^{3}\left(\mathbb{D}\right.$, Hassan Algadi $^{2,4}{ }^{\mathbb{D}}$, Ahmed A. Ibrahim $^{1,2}$, Mohsen A. M. Alhamami ${ }^{1}$ \\ and Sotirios Baskoutas $5, *$ (D) \\ 1 Department of Chemistry, Faculty of Science and Arts, Najran University, P.O. Box 1988, Najran 11001, \\ Saudi Arabia; ahmedragal@yahoo.com (A.A.I.); maalhammami@nu.edu.sa (M.A.M.A.) \\ 2 Promising Centre for Sensors and Electronic Devices (PCSED), Najran University, P.O. Box 1988, \\ Najran 11001, Saudi Arabia; hassan.algadi@gmail.com \\ 3 New \& Renewable Energy Material Development Center (NewREC), Jeonbuk National University, Jeonju-si, \\ Jeollabuk-do 54896, Korea; shaheerakhtar@jbnu.ac.kr \\ 4 Department of Electrical Engineering, Faculty of Engineering, Najran University, P.O. Box 1988, Najran 11001, \\ Saudi Arabia \\ 5 Department of Materials Science, University of Patras, GR-26504 Patras, Greece \\ * Correspondence: ahmadumar786@gmail.com or umahmad@nu.edu.sa (A.U.); bask@upatras.gr (S.B.)
}

check for updates

Citation: Umar, A.; Akhtar, M.S.; Algadi, H.; Ibrahim, A.A.; Alhamami, M.A.M.; Baskoutas, S. Highly Sensitive and Selective Eco-Toxic 4-Nitrophenol Chemical Sensor Based on Ag-Doped ZnO Nanoflowers Decorated with Nanosheets. Molecules 2021, 26, 4619. https:// doi.org/10.3390/molecules26154619

Academic Editor: Eugene L Kolychev

Received: 24 June 2021

Accepted: 20 July 2021

Published: 30 July 2021

Publisher's Note: MDPI stays neutral with regard to jurisdictional claims in published maps and institutional affiliations.

Copyright: (c) 2021 by the authors. Licensee MDPI, Basel, Switzerland. This article is an open access article distributed under the terms and conditions of the Creative Commons Attribution (CC BY) license (https:// creativecommons.org/licenses/by/ $4.0 /)$.

\begin{abstract}
Herein, we have developed a novel sensing electrode to detect the eco-toxic 4-nitrophenol (4-NP). Ag-doped-ZnO nanoflowers were synthesized by facile hydrothermal method and examined by several characterization techniques in order to understand the morphology, crystal structure, composition, and surface properties. Morphological results were confirmed by the formation of Ag-doped $\mathrm{ZnO}$ nanoflowers decorated with nanosheets. Ag-doped $\mathrm{ZnO} /$ glassy carbon electrode (GCE) electrode-material-matrix was used for electrochemical sensing of toxic 4-NP. Under optimized conditions, Ag-doped $\mathrm{ZnO} / \mathrm{GCE}$ modified electrode exhibits high-sensitivity and selectivity compared to the bare GCE electrode. The Ag-doped ZnO/GCE modified electrode exhibits high electrocatalytic oxidation towards 4-NP. Anodic peak current of 4-NP is increased linearly by increasing the concentration of nitrophenol. Additionally, Ag-doped $\mathrm{ZnO} / \mathrm{GCE}$ shows a wide range of sensitivity from $10 \mu \mathrm{M}$ to $500 \mu \mathrm{M}$, and a linear calibration plot with a good detection limit of $3 \mu \mathrm{M}$ (S/N = 3). The proposed Ag-doped ZnO/GCE modified electrode showed high sensing stability. In addition, the oxidation mechanism was studied. The obtained results revealed that the Ag-ZnO/GCE electrode could be the promising sensing electrode for 4-NP sensing.
\end{abstract}

Keywords: Ag-doped $\mathrm{ZnO}$; nanoflowers; electrochemical sensor; 4-nitrophenol; oxidation

\section{Introduction}

Detecting nitro aromatic compounds is extraordinarily significant in assessing the danger of various natural tests such as wastewater from mining, paint and drug enterprises, petrochemical items, or the assembling of pesticides [1-5], which have a tendency to damage the living organism and plants in low concentrations [6]. Mainly, nitrophenol is formed during the hydrolysis process of diethyl parathion, which is reported by the United State Environmental Protection Agency (USEPA). Nitrophenol is a common intermediate product used for the production of analgesic, dyes, medicines, leather products, and so on. It is considered a hazardous pollutant in food chains such as fruits, and cultivation crops [7-10]. Nitrophenol compounds also cause severe environmental pollution, owing to their highly toxic level for humans and animals, and thus have been included in the list of toxic and harmful pollutants in different countries. Because of this, more sensitive and accurate detection of nitrophenol is important and crucial [11]. 
Several different methods were used for efficient detection of nitro compounds, to name a few, fluorescence, capillary zone electrophoresis, spectrophotometry, HPLC, electrochemical analysis, and so on [12-16]. Among all the methods, the electrochemical analysis is given more consideration due to its outstanding sensitivity, selectivity, cost effective and easy operation techniques [17]. Still, several reports demonstrated oxidation and reduction of nitrophenol with different electrode materials such as carbon materials (carbon nanotubes, graphene, carbon dots, etc.), mesoporous and microporous carbon graphene, metal and metal oxide nanomaterials and their composites, metal sulphides and so on [18-21]. Nevertheless, the majority of researchers focused on the development of electrode materials for better electrocatalytic activity for nitrophenol detection with high selectivity and sensitivity. However, the metallic nanoparticles had outstanding electrical conductivity and catalytic activity towards chemical processes. Gold nanoparticles have a smaller particle size, better electrical conductivity and outstanding surface roughness. However, the scarcity and cost of gold nanoparticles limits the practical applications. Hence, the development of a novel and low-cost sensing material is highly desirable [22].

In this paper, we report the synthesis of Ag-doped $\mathrm{ZnO}$ nanoflowers decorated with nanosheets and their sensing ability of toxic 4-nitrophenol. The synthesized material was examined using several techniques in order to understand the morphology, crystal structure, composition, and surface properties. Ag-doped $\mathrm{ZnO}$ nanoflowers decorated with nanosheets were used as an electrode-material-matrix to fabricate electrochemical sensors which exhibited high-sensitivity and selectivity towards 4-nitrophenol. Under optimized conditions, the Ag-doped $\mathrm{ZnO} / \mathrm{GCE}$ modified electrode exhibits high-sensitivity and selectivity compared to the bare GCE electrode. Additionally, Ag-doped ZnO/GCE showed a wide range of sensitivity from $10 \mu \mathrm{M}$ to $500 \mu \mathrm{M}$ and a linear calibration plot with a good detection limit of $3 \mu \mathrm{M}(\mathrm{S} / \mathrm{N}=3)$. In addition, the oxidation mechanism was studied. The obtained results revealed that the Ag-ZnO/GCE electrode could be the promising sensing electrode for 4-NP sensing.

\section{Results and Discussion}

2.1. Characterizations and Properties of Ag-Doped $\mathrm{ZnO}$ Nanoflowers Decorated with Nanosheets

Crystal structure, planes, and phases of the produced Ag-doped $\mathrm{ZnO}$ nanoflowers decorated with nanosheets were determined using the $X$-ray diffraction technique. Figure 1 exhibits the typical XRD pattern of as-synthesized Ag-doped $\mathrm{ZnO}$ nanoflowers decorated with nanosheets. Several well-defined diffraction peaks were identified in the observed diffraction pattern, which were closely associated with the wurtzite hexagonal phase of $\mathrm{ZnO}$. The diffraction peaks seen at $2 \theta=31.7^{\circ}, 34.3^{\circ}, 36.3^{\circ}, 47.5^{\circ}, 56.4^{\circ}, 62.9^{\circ}, 66.5^{\circ}, 68.0^{\circ}$, $69.1^{\circ}, 72.6^{\circ}$ and $77.1^{\circ}$ can be assigned to various diffraction planes of $\mathrm{ZnO}(100)$, (002), (101), (102), (110), (103), (200), (112), (201), (004) and (202), respectively. All the observed diffraction peaks were consistent with the JCPDS card no. 36-1451 and reported literature related to the wurtzite hexagonal phase of $\mathrm{ZnO}$ [23-25]. Furthermore, two other diffraction peaks appeared at $2 \theta=38.1^{\circ}$ and $44.3^{\circ}$ which can be assigned to diffraction planes of $\mathrm{Ag}$ (111) and (200), respectively, and exhibit the Ag face-centered-cubic (fcc) crystal structure. The observed Ag peaks are consistent with the reported literature and JCPDS data card no. 04-0783 [26,27]. 


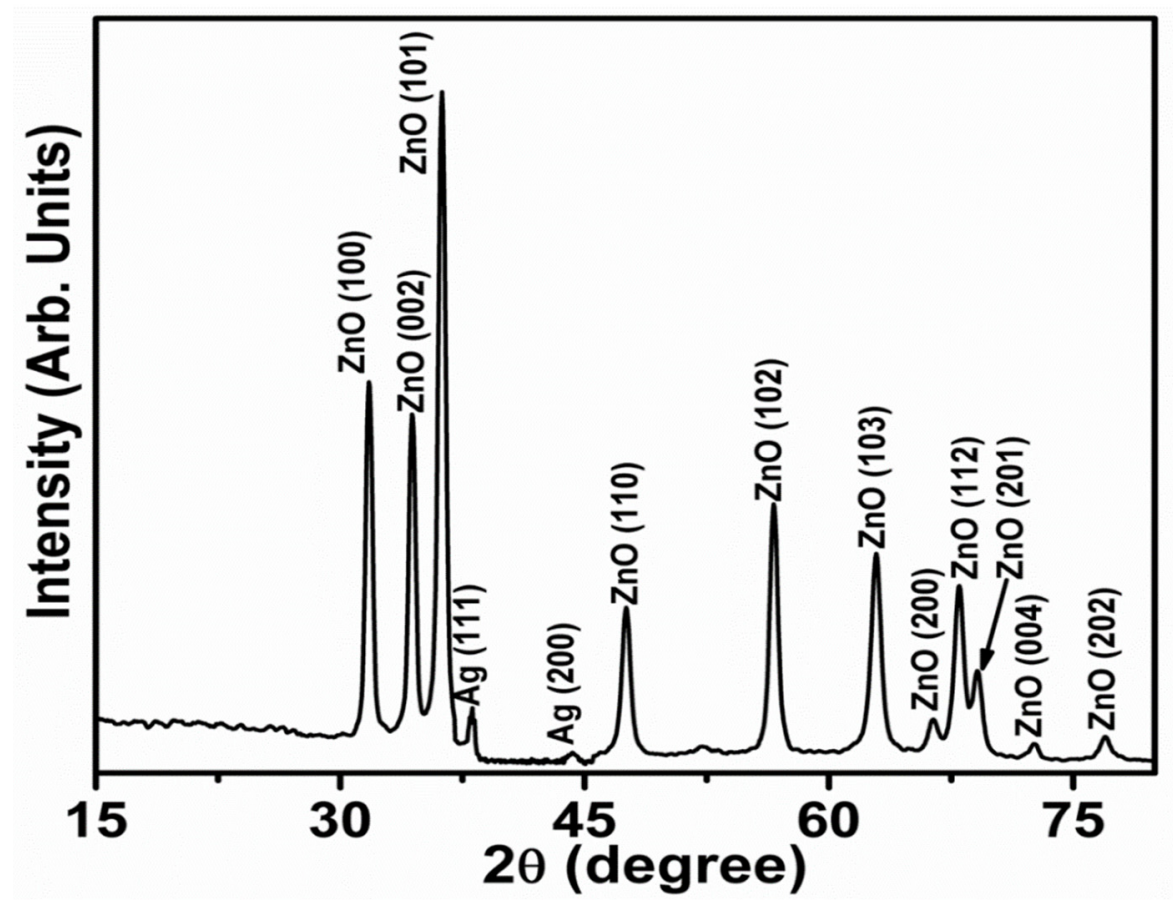

Figure 1. Powder X-ray diffraction pattern of Ag-doped $\mathrm{ZnO}$ nanoflowers.

Surface morphology of the Ag-doped $\mathrm{ZnO}$ nanomaterial was scrutinized by FESEM. The findings are displayed in Figure 2. The synthesized material had flower-shaped morphologies, grown in very high density, as evidenced by low-magnification FESEM images (Figure 2a-c). The flower-shaped morphologies were composed of well-defined nanorods which seem to originate from a single nucleus (Figure $2 d-f$ ). The hexagonal nanorods possessed wider bases and narrower tips. The typical lengths and diameters of the nanorods were in the range of $6 \pm 1 \mu \mathrm{m}$ and $150 \pm 30 \mathrm{~nm}$, respectively.

Interestingly, it was observed that the hexagonal nanorods were decorated with sheetlike morphologies (Figure 2e,f). The thickness of the nanosheets was around 10-15 nm. The nanosheets were precisely wrapped on the nanorods' surfaces. The typical size of a single flower-shaped structure was $10 \pm 2 \mu \mathrm{m}$.

The elemental composition of the synthesized Ag-doped $\mathrm{ZnO}$ nanoflowers decorated with nanosheets was examined by energy dispersive spectroscopy and elemental mapping. Figure 3a depicts the typical SEM and $(\mathrm{b}-\mathrm{d})$ their corresponding elemental mapping images. The EDS spectrum of synthesized Ag-doped $\mathrm{ZnO}$ nanoflowers is shown in the inset of Figure 3a, confirming that the material is only composed of zinc ( $\mathrm{Zn})$, oxygen $(\mathrm{O})$, and silver. There were no other peaks in the EDS spectrum were related to any other element, confirming that the material is made of $\mathrm{Zn}, \mathrm{O}$, and $\mathrm{Ag}$ only, without other elements. Figure $3 \mathrm{~b}-\mathrm{d}$ exhibits the elemental mapping of Ag-doped $\mathrm{ZnO}$ flower shaped structures. It is clear from the observed elemental mapping that the number of spots corresponding to $\mathrm{O}$ and $\mathrm{Zn}$ are homogeneous and higher in number over the whole structure, confirmed by the uniform distribution of $\mathrm{Zn}$ and O over the whole crystals of flower-shaped structures (Figure $3 b, c$ ). The low number of spots in the elemental scan of $\mathrm{Ag}$ in the corresponding flower-shaped morphology revealed that Ag is well doped into the lattices of the synthesized material (Figure 3d). Hence, the observed elemental mapping results proved the formation of Ag-doped $\mathrm{ZnO}$. 

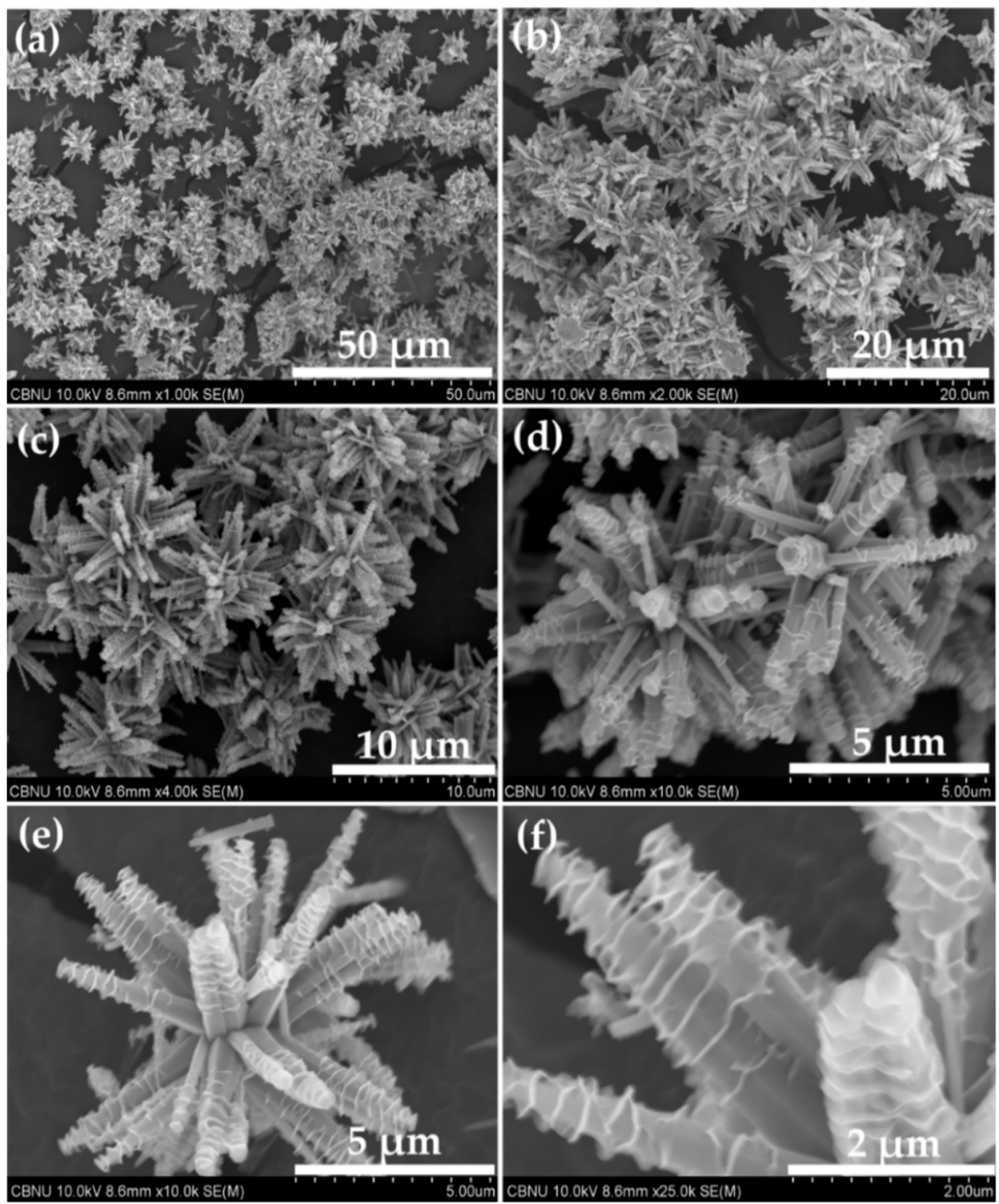

Figure 2. FESEM micrographs of synthesized Ag-doped $\mathrm{ZnO}$ nanoflowers decorated with nanosheets.

Further detailed morphology and structural features of synthesized Ag-doped $\mathrm{ZnO}$ nanoflowers decorated with nanosheets were monitored by HR-TEM. The synthesized material was dispersed in acetone and ultrasonicated for 5 min for TEM examination. Consequently, a drop of acetone containing the synthesized material was placed on the TEM grid and examined. The flower-shaped morphology was torn down into nanorods with ultrasonication. Figure 4a depicts the typical TEM image of a single nanorod. The morphology of the nanorod is consistent with the observed FESEM results, i.e., a sharpened tip with a wider base. Interestingly, the nanorod showed the decoration of nanosheets over its surface. The HR-TEM image, obtained from the circled portion of a single nanorod shown in Figure 4a, clearly displayed well-defined lattice fringes with a d-spacing value between two fringes of $0.26 \mathrm{~nm}$, corresponding to the interplanar spacing of the (0002) plane of wurtzite hexagonal phase $\mathrm{ZnO}$ (Figure 4b) [28]. The well-defined lattice fringes confirmed the formation of well-crystalline Ag-doped $\mathrm{ZnO}$ nanoflowers. 


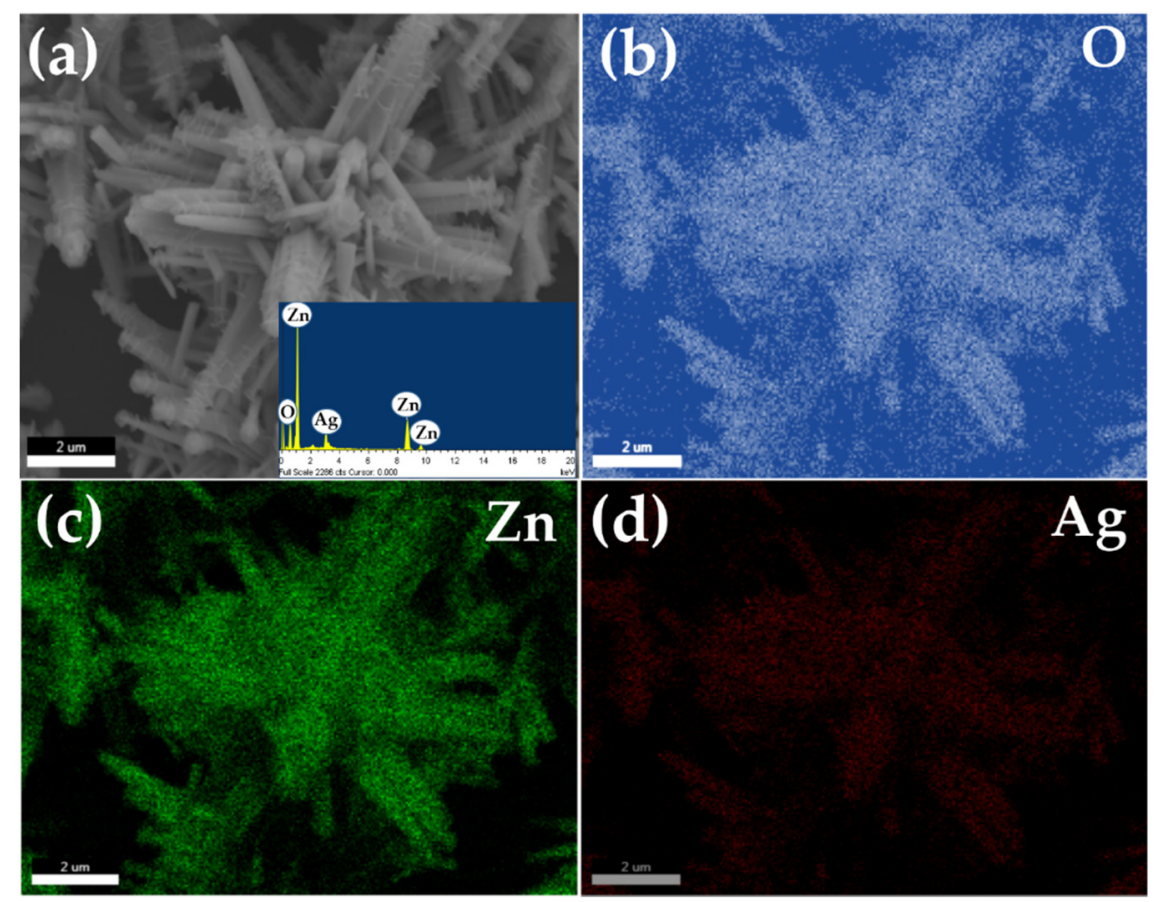

Figure 3. Typical (a) SEM image (inset: EDS spectrum) (b-d) O, Zn and Ag elemental mapping of synthesized Ag-doped $\mathrm{ZnO}$ nanoflowers.
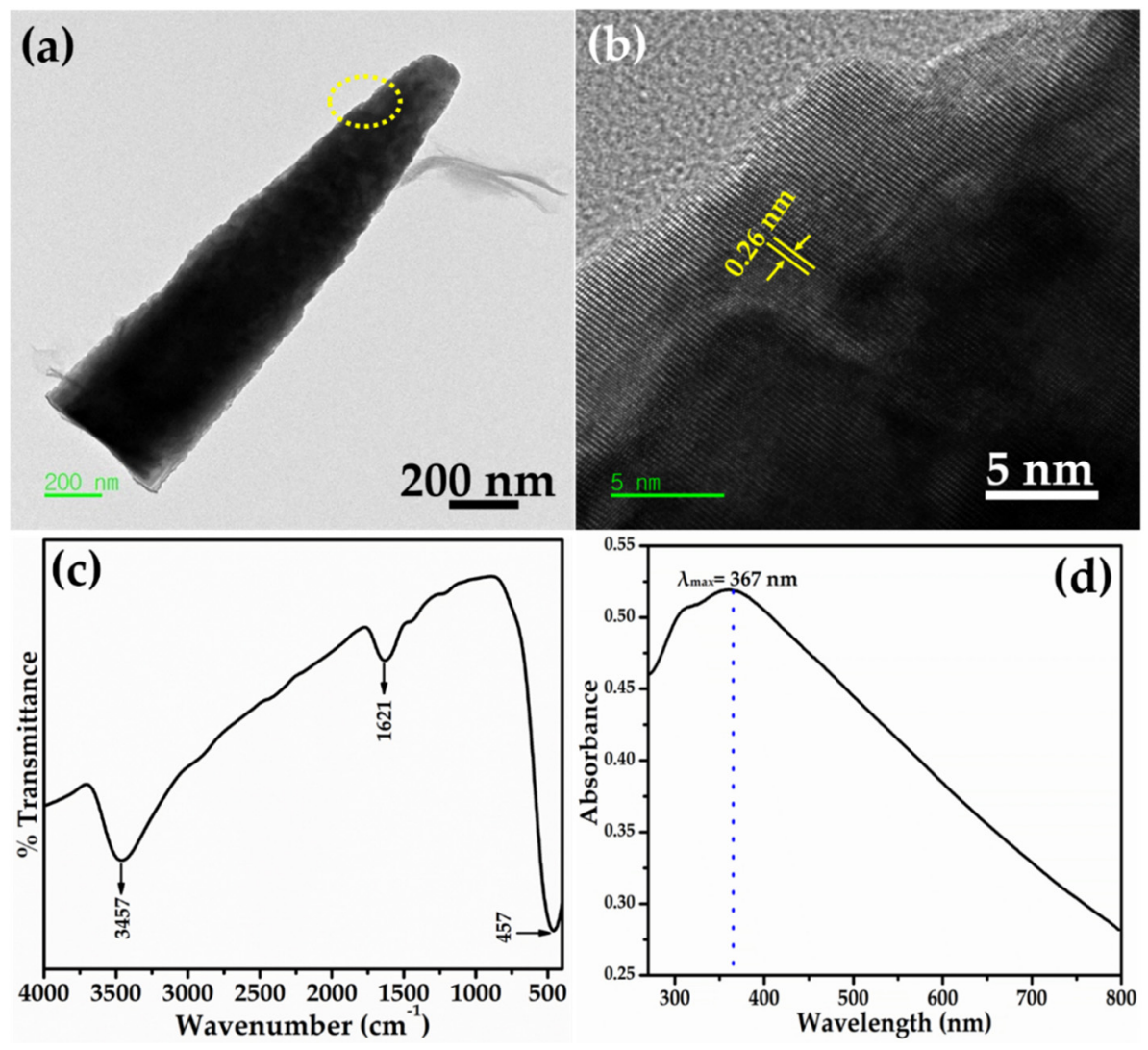

Figure 4. (a) HR-TEM image, (b) lattice fringes, (c,d) FTIR and UV-visible spectra of Ag-doped ZnO nanoflowers decorated with nanosheets.

The metal bonding and functional groups of the Ag-doped $\mathrm{ZnO}$ nanoflowers decorated with nanosheets was measured using Fourier transform infrared (FTIR) spectroscopy. 
Figure 4c demonstrates the typical FTIR spectrum of synthesized Ag-doped $\mathrm{ZnO}$ nanoflowers. Few well-defined peaks are observed in the FTIR spectrum at $457 \mathrm{~cm}^{-1}, 1621 \mathrm{~cm}^{-1}$, and $3457 \mathrm{~cm}^{-1}$. The band at $457 \mathrm{~cm}^{-1}$ corresponded to the metal-oxygen (M-O) stretching vibration, confirming the formation of Ag-ZnO nanomaterial [28]. The water O-H stretching and bending frequency was observed at a higher frequency which, due to absorbed water molecules on the surface of the $\mathrm{Ag}$-doped $\mathrm{ZnO}$ nanoflowers, may be responsible for the well-defined IR bands at $1621 \mathrm{~cm}^{-1}$ and $3457 \mathrm{~cm}^{-1}$ [29].

Figure $4 \mathrm{~d}$ shows the UV-visible spectrum of synthesized Ag-doped $\mathrm{ZnO}$ nanoflowers. An intense absorption peak was observed at $367 \mathrm{~nm}$. The band gap energy was calculated according to Equation (1):

$$
E_{g}=\frac{1240}{\lambda \max }
$$

According to Equation (1), the calculated band gap energy of the synthesized Agdoped $\mathrm{ZnO}$ nanoflowers was $3.38 \mathrm{eV}$, within the ideal band gap of wurtzite hexagonal phase $\mathrm{ZnO}[30,31]$.

The chemical compositions and oxidation states of Ag-doped $\mathrm{ZnO}$ nanoflowers were studied using X-ray photoelectron spectroscopy (XPS), as shown in Figure 5. To avoid surface charging, all binding energies were corrected using the referenced neutral carbon peak C 1s at $284.8 \mathrm{eV}$. Doublet binding energies appeared at 1020.9 and $1044.1 \mathrm{eV}$, which connect to $\mathrm{Zn} 2 \mathrm{p}_{3 / 2}$ and $\mathrm{Zn} 2 \mathrm{p}_{1 / 2}$ spin splitting, respectively, in the high-resolution $\mathrm{Zn}$ $2 p$ XPS spectrum (Figure 5a). The spin splitting difference of the $Z n 2 p_{1 / 2}$ and $Z n 2 p_{3 / 2}$ peaks was estimated to be $23.2 \mathrm{eV}$ for Ag-doped $\mathrm{ZnO}$ nanoflowers, which is close to $\mathrm{Zn}^{2+}$ in $\mathrm{ZnO}$ [32]. Figure $5 \mathrm{~b}$ depicts the resolved Ag $3 \mathrm{~d}$ XPS spectrum, which demonstrates that doublet binding energies appeared at 374.9 and $368.7 \mathrm{eV}$, corresponding to the $\mathrm{Ag}$ $3 \mathrm{~d}_{3 / 2}$ and $\mathrm{Ag} 3 \mathrm{~d}_{5 / 2}$ spin splitting, respectively. The presence of $A g 3 \mathrm{~d}_{5 / 2}$ with a binding energy of $368.7 \mathrm{eV}$ is related to the metallic Ag; however, it is closer to the binding energy of $\mathrm{Ag}+$ (i.e., $367.9 \mathrm{eV}$ ), clearly indicating the presence of $\mathrm{Ag}+$ in $\mathrm{ZnO}$ [33]. Because of the valence state difference between $\mathrm{Ag}^{+}$and $\mathrm{Zn}^{2+}$ ions, the replacement of $\mathrm{Ag}^{+}$at $\mathrm{Zn}^{2+}$ sites in $\mathrm{ZnO}$ crystals tends to form zinc interstitial $\left(\mathrm{Zn}_{\mathrm{i}}\right)$ or $\mathrm{Vo}$. This caused a reduction in the real doping amount of $\mathrm{Ag}^{+}$, which reduced the $\mathrm{Zn}_{\mathrm{i}}$ or Vo contents. The resolved $\mathrm{O} 1 \mathrm{~s}$ XPS spectrum was analyzed in order to understand the oxide bonding in the synthesized material. The observed O1s spectrum exhibited two fitted XPS peaks with binding energies of 529.2 and $531.8 \mathrm{eV}$ (Figure 5c). The peak at $529.2 \mathrm{eV}$ could be related to the $\mathrm{O}^{2-}$ ions in $\mathrm{Zn}-\mathrm{O}$, revealing the formation of $\mathrm{ZnO}$ in $\mathrm{Ag}$-doped $\mathrm{ZnO}$, while the peak at $531.8 \mathrm{eV}$ can be attributed to the adsorbed moisture over the materials surface, which might generate structural defects $[34,35]$. 

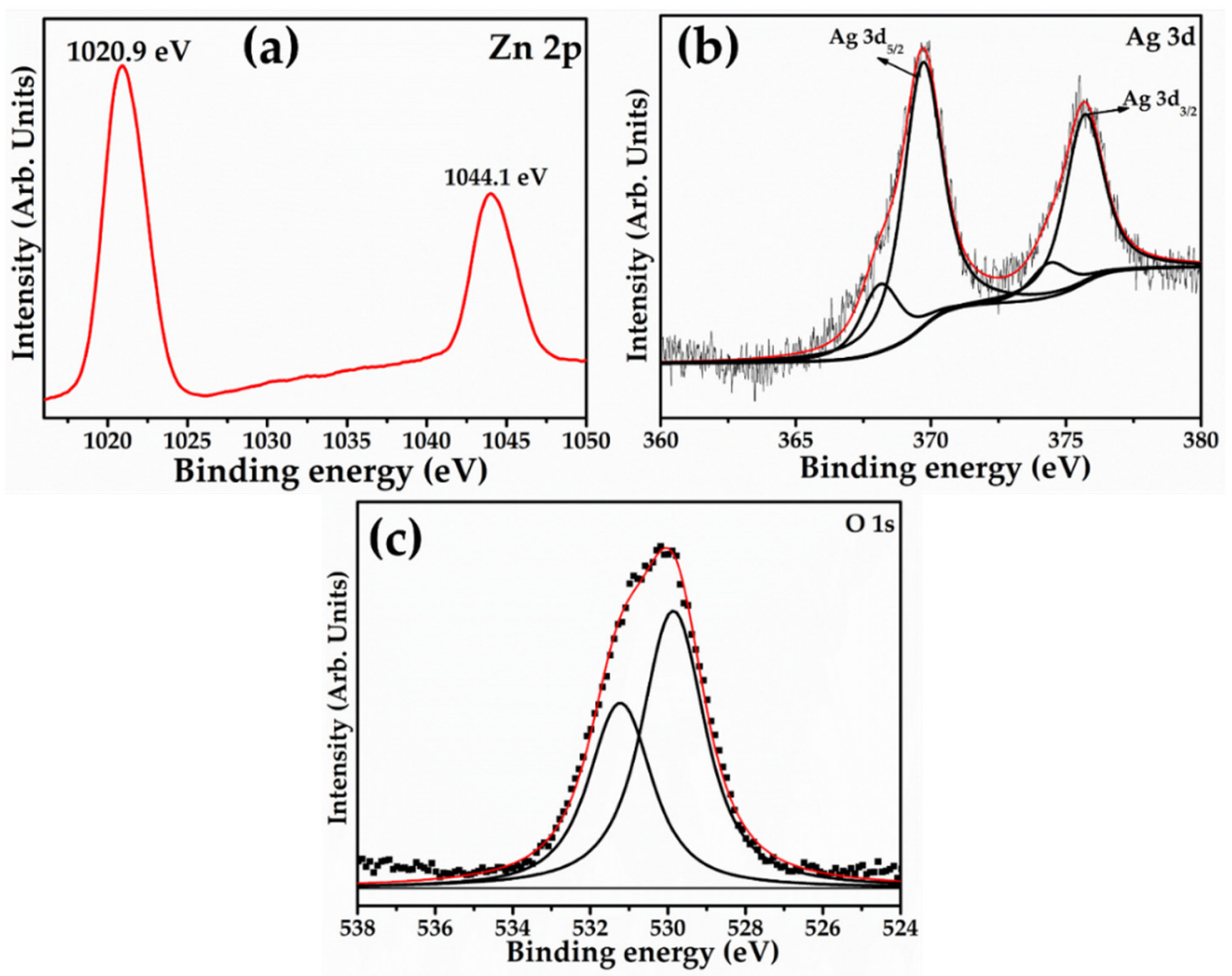

Figure 5. Typical XPS spectra of (a) Zn 2p, (b) Ag 3d, and (c) O 1s of synthesized Ag-doped ZnO nanoflowers decorated with nanosheets.

\subsection{Electrochemical Activity of Ag-ZnO/GCE Electrode}

Electrochemical activity of the Ag-ZnO/GCE modified electrode was analyzed using cyclic voltammetry technique. Briefly, Ag- $\mathrm{ZnO}$ modified glassy carbon electrode (Ag$\mathrm{ZnO} / \mathrm{GCE}$ ) was tested with $5 \mathrm{mM}$ of ferri-ferro redox couple in $0.1 \mathrm{M} \mathrm{KCl}$ solution. Figure 6a demonstrated the standard ferri-ferro redox couple of GCE and Ag-ZnO/GCE electrodes. The bare GCE electrode showed the defined redox peaks with the $60 \mu \mathrm{A}$ peak current, whereas the Ag-ZnO/GCE modified electrode showed a well-defined reversible redox couple with high anodic and cathodic peak current. This was due to the high electron transfer kinetics between the electrode and electrolyte. Moreover, the Ag doping enhanced the electron transfer rate, and the effect of rate variation $\left(10 \mathrm{mV} \mathrm{s}^{-1}\right.$ to $\left.200 \mathrm{mV} \mathrm{s}^{-1}\right)$ on the electrical activity of the Ag-ZnO/GCE electrode in ferri-ferro solution (Figure 6b). The obtained results revealed that redox peak current linearly increased by increasing the scan rate as shown in Figure 6c. Furthermore, electrochemical impedance spectroscopy (EIS) was used to monitor the resistance of the Ag-ZnO-GCE electrode in ferri-ferro solution.

Figure $6 \mathrm{~d}$ shows the Nyquist plot of the GCE and Ag-ZnO/GCE electrode. The bare GCE electrode showed a higher resistance $R_{\mathrm{ct}}$ value of $2 \mathrm{k} \mathrm{ohm}$; after the modification with $\mathrm{Ag}-\mathrm{ZnO}$ nanoflowers, it showed a lower resistance value of $0.5 \mathrm{k} \mathrm{ohm}$, which confirms the higher electron transfer capacity of the Ag-ZnO/GCE electrode. Hence, the EIS results revealed that $\mathrm{Ag}-\mathrm{ZnO} / \mathrm{GCE}$ is the convenient electrode for electrochemical sensing applications. 

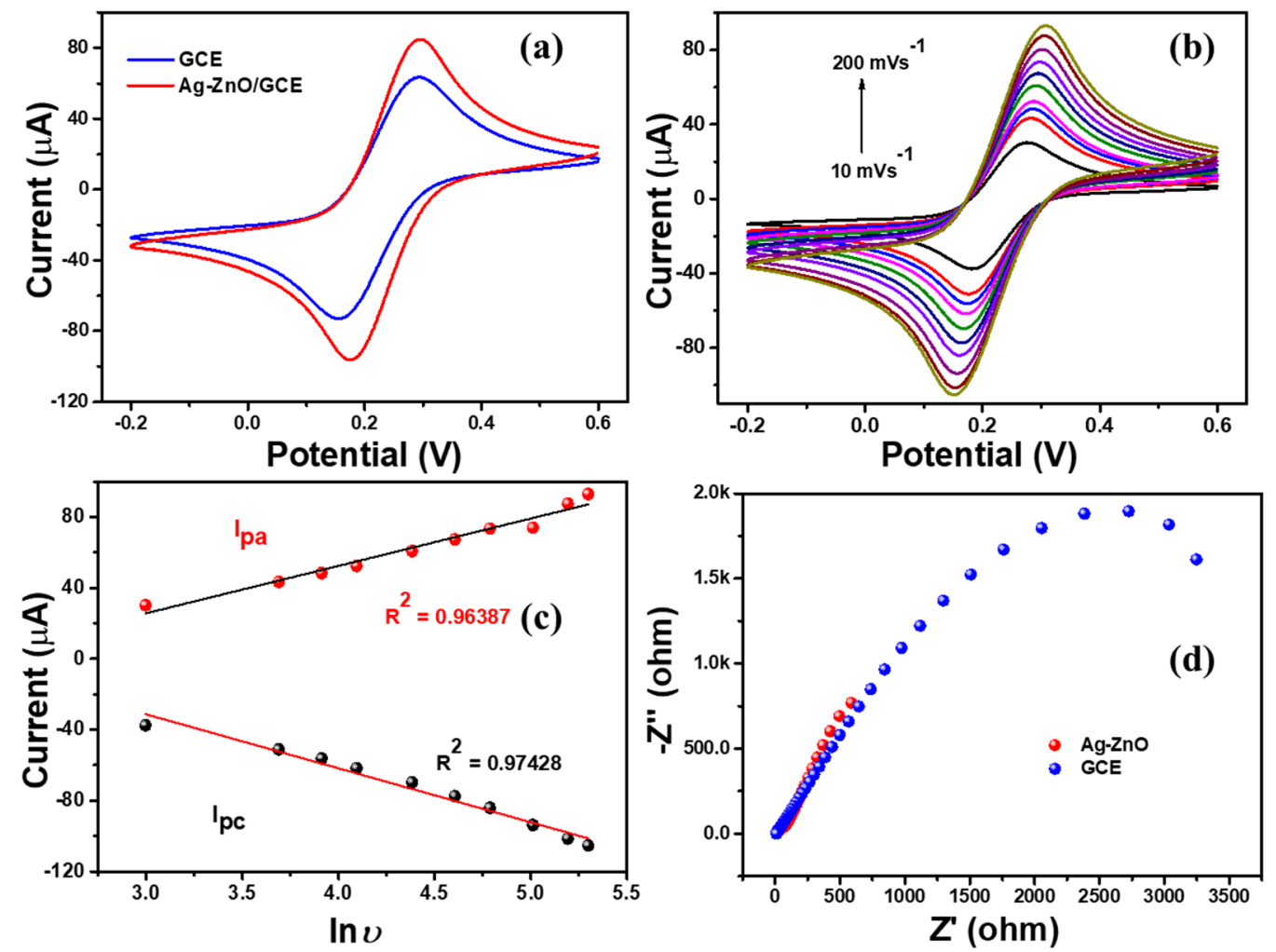

Figure 6. (a) CV responses of bare GCE and Ag-ZnO/GCE, (b) scan rate variation of Ag-ZnO/GCE, (c) linear regression plot (d) Nyquist plot for bare GCE and Ag-ZnO/GCE electrode with $5 \mathrm{mM} \mathrm{K}_{3}$ $\left[\mathrm{Fe}(\mathrm{CN})_{6}\right]$ in $0.1 \mathrm{M} \mathrm{KCl}$ electrolyte.

\subsection{Sensing Applications of Ag-Doped ZnO Nanoflowers}

The electrochemical sensing activity of bare GCE and Ag-ZnO/GCE electrode was investigated with $0.5 \mu \mathrm{M} 4-\mathrm{NP}$ containing $0.1 \mathrm{M}$ PBS solution at a scan rate of $100 \mathrm{mV} \mathrm{s}^{-1}$. Figure 7a shows the CV responses of bare GCE and Ag-ZnO/GCE with $500 \mu \mathrm{M} 4-\mathrm{NP}$ containing 0.1 M PBS solution. Bare GCE showed the weak oxidation peak of 4-NP at $0.9 \mathrm{~V}$ with poor peak current, which confirms the poor catalytic activity of the bare GCE electrode [36,37].
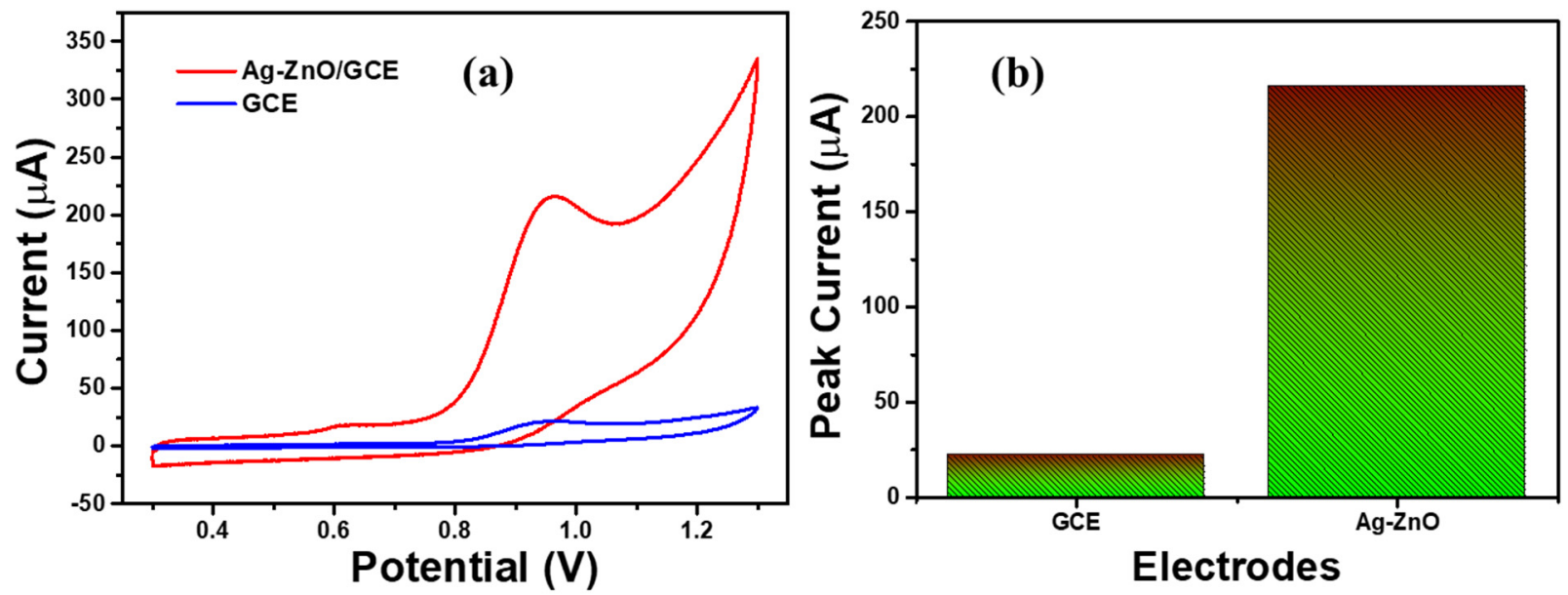

Figure 7. (a) CV responses of bare GCE and Ag-ZnO/GCE (b) 4-NP sensing activity of the bare GCE and Ag-ZnO/GCE electrode with presence of $500 \mu \mathrm{M}$ 4-NP containing $0.1 \mathrm{M}$ PBS $(\mathrm{pH}=7)$ solution. (Accumulation time: 2 min, catalyst loading volume: $10 \mu \mathrm{L}$ ). 
The Ag-ZnO/GCE electrode showed well defined oxidation peak of 4-NP with huge peak current. This confirms the excellent catalytic activity toward 4-NP sensing. In addition, the 4-NP peak current of both electrodes was plotted, and is shown in Figure 7b. This showed excellent sensing activity due to the synergistic of the Ag-doped $\mathrm{ZnO}$ nanoflowers. Furthermore, sensitivity of the Ag- $\mathrm{ZnO} / \mathrm{GCE}$ electrode was analyzed with different concentrations ranging from $10 \mu \mathrm{M}$ to $500 \mu \mathrm{M}$ 4-NP solution in $0.1 \mathrm{M}$ PBS solution as shown in Figure 8 . The oxidation peak current linearly increased with respect to the concentration of 4-NP. In addition, the calibration plot was fitted against the peak current vs. concentration of 4-NP. This confirmed the sensitivity of Ag-ZnO/GCE towards 4-NP in neutral medium.
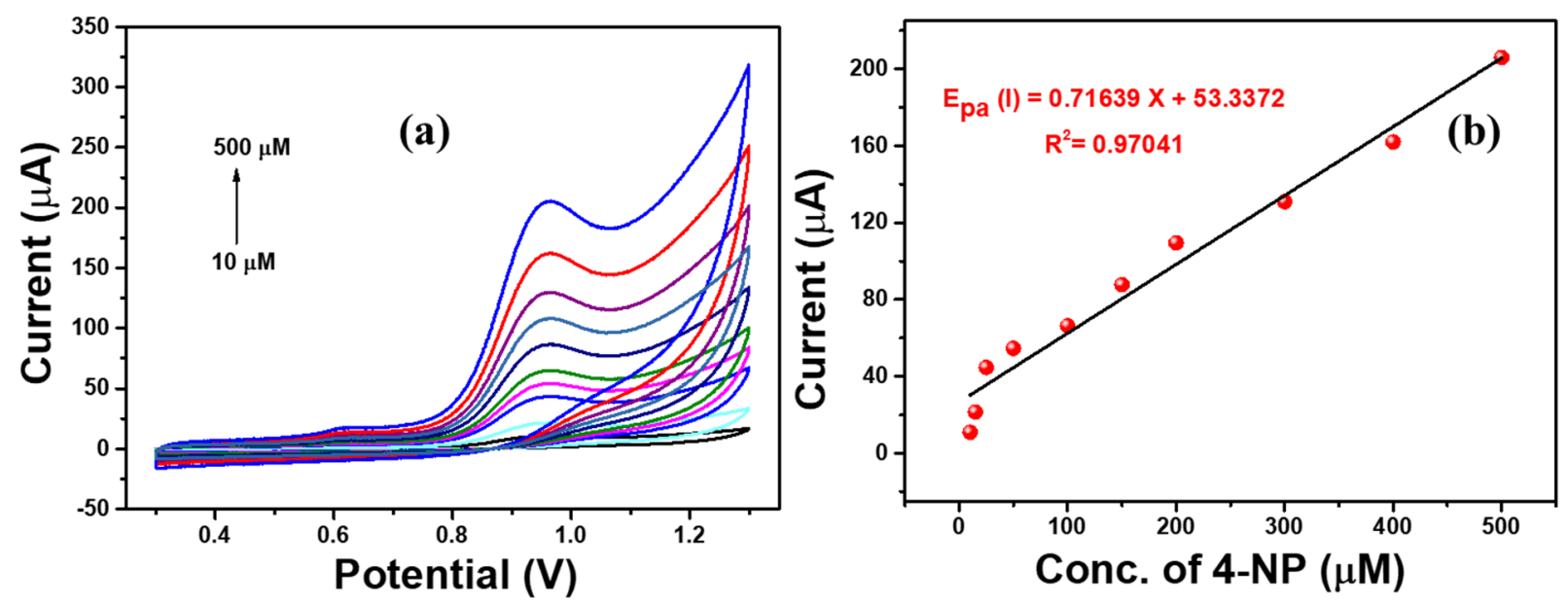

Figure 8. (a) CV responses of Ag-ZnO/GCE at different 4-NP concentrations (10 $\mu \mathrm{M}$ to $500 \mu \mathrm{M})$ containing $0.1 \mathrm{M}$ PBS $(\mathrm{pH}=7)$ solution at a scan rate of $100 \mathrm{mV} \mathrm{s}^{-1}$. (b) Linear regression plot of current vs. 4-NP concentration. (Accumulation time: 2 min, catalyst loading volume: $10 \mu \mathrm{L}$ ).

In addition, the effect of the scan rate on the 4-NP sensing of Ag-ZnO/GCE was analyzed by varying the applied scan rate from $10 \mathrm{mV} \mathrm{s}^{-1}$ to $200 \mathrm{mV} \mathrm{s}^{-1}$ with the presence of $500 \mu \mathrm{M} 4-\mathrm{NP}$ containing $0.1 \mathrm{M}$ PBS $(\mathrm{pH}=7)$ solution. Figure 9 shows that the oxidation peak current increased linearly with increasing scan rate. In addition, the peak current was plotted against the scan, is shown in Figure 9b, which suggested that the 4- NP oxidation is a diffusion-controlled process $[38,39]$.
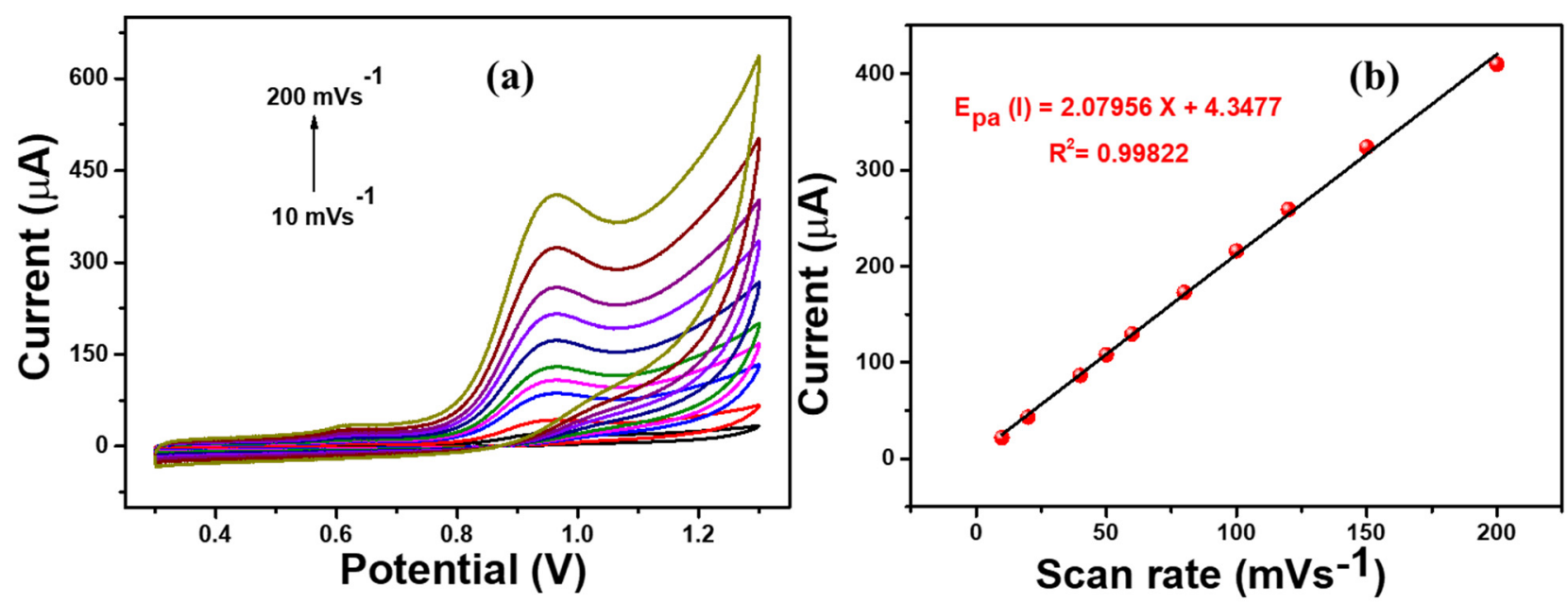

Figure 9. (a) $\mathrm{CV}$ responses of $\mathrm{Ag}-\mathrm{ZnO} / \mathrm{GCE}$ at different scan rate variations $\left(10 \mathrm{mV} \mathrm{s}^{-1}\right.$ to $\left.200 \mathrm{mV} \mathrm{s}^{-1}\right)$, (b) linear regression plot of current vs. scan rate in presence of $500 \mu \mathrm{M} 4-\mathrm{NP}$ containing $0.1 \mathrm{M}$ PBS $(\mathrm{pH}=7)$ solution. (Accumulation time: 2 min, catalyst loading volume: $10 \mu \mathrm{L}$ ). 


\subsection{Linear Sweep Voltammetry (LSV) Analysis of 4-NP}

Linear Sweep Voltammetry (LSV) analysis is a simple and sensitive technique to sense the 4-NP over the Ag-ZnO/GCE-modified electrode. It is a simple and time saving technique due to its controllable sweep direction. Hence, we used the LSV technique for electrochemical sensing of 4-nitrophenol. The LSV responses were observed at different concentrations of 4-NP $(10 \mu \mathrm{M}$ to $500 \mu \mathrm{M})$ in $0.1 \mathrm{M}$ PBS solution at a scan rate of $100 \mathrm{mV} \mathrm{s}^{-1}$. Figure 10 shows that the 4-NP oxidation peak current increased linearly by increasing the concentration of nitrophenol. In addition, the calibration was plotted with peak current against 4-NP concentration and is shown in Figure 10b. The Ag-ZnO modified electrode showed an excellent linear regression equation $\left(R^{2}=0.97206\right)$.
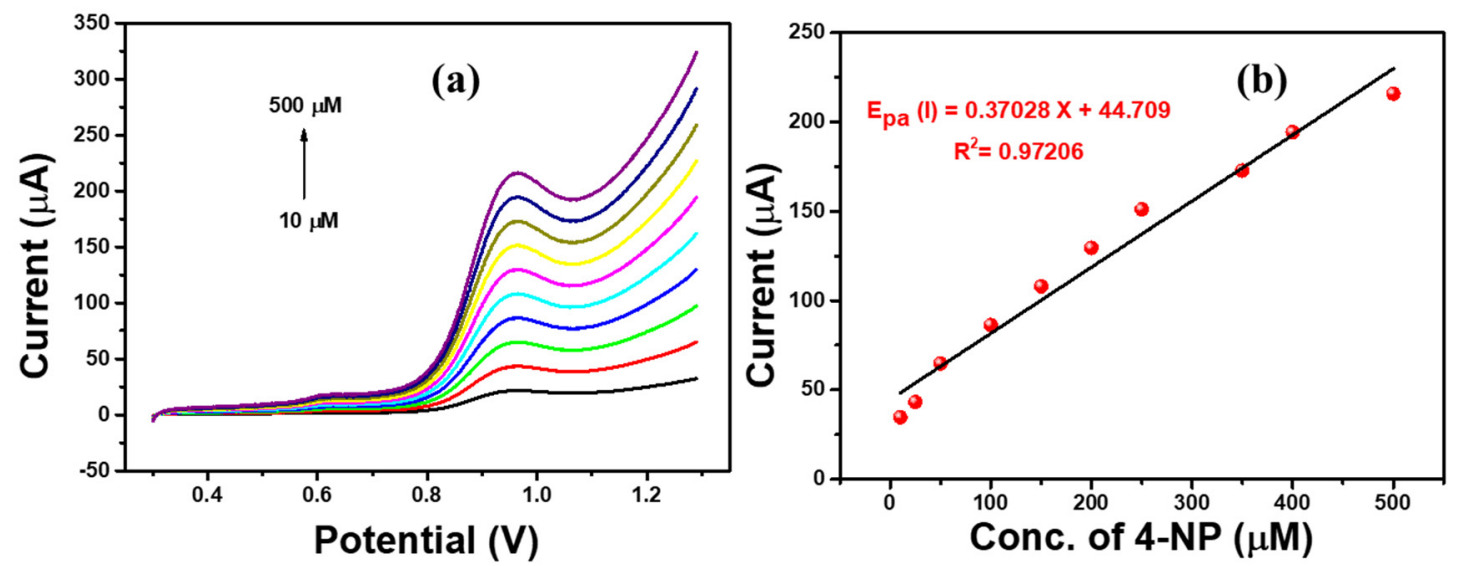

Figure 10. (a) LSV responses of Ag-ZnO/GCE at different 4-NP concentrations (10 $\mu \mathrm{M}$ to $500 \mu \mathrm{M})$ containing $0.1 \mathrm{M}$ PBS $(\mathrm{pH}=7)$ solution at a scan rate of $100 \mathrm{mV} \mathrm{s}^{-1}$. (b) Linear regression plot of current vs. 4-NP concentration. (Accumulation time: 2 min, catalyst loading volume: $10 \mu \mathrm{L}$ ).

Furthermore, the low detection limit, $3 \mu \mathrm{M}(\mathrm{S} / \mathrm{N}=3)$, was derived for $\mathrm{Ag}-\mathrm{ZnO} / \mathrm{GCE}$ electrode which revealed that the Ag-ZnO/GCE electrode could be the promising sensing electrode for 4-NP sensing. In addition, the oxidation mechanism was studied and is shown in Scheme 1. Generally, 4-NP oxidation followed an irreversible reaction at solution $\mathrm{pH}$ 7, which is a neutral medium. The 4-NP oxidation mechanism followed through phenolate radical formation and the results suggested that 4-nitrophenol oxidized to 4-nitrosophenol through phenolate radical formation which confirms the formation of intense oxidation peak at $0.9 \mathrm{~V}$.

\section{4-nitrophenol}

4-nitrosophenol

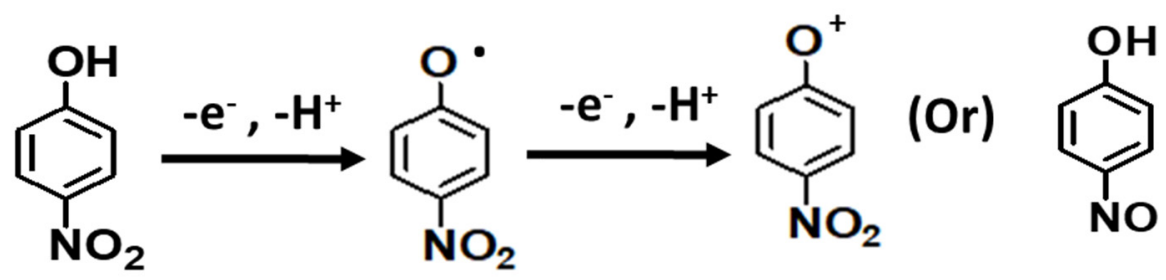

Scheme 1. 4-NP oxidation mechanism on the Ag-ZnO/GCE electrode.

Generally, repeatability and stability of the sensor electrode was the key point in the electrochemical sensing application. Thus we analyzed the sensing stability of the Ag-ZnO/GCE electrode with $500 \mu \mathrm{M}$ 4-NP containing $0.1 \mathrm{M} \mathrm{PBS}(\mathrm{pH}=7)$ solution at a scan rate of $100 \mathrm{mV} \mathrm{s}^{-1}$ for more than 5 weeks and the obtained results are shown in Figure 11. The Ag-ZnO/GCE electrode showed good sensing stability towards 4-NP over 5 weeks. 
From Figure $11 \mathrm{~b}$ it was observed that, the peak current slightly decreased over three weeks. Hence, Ag-ZnO/GCE electrode could be the promising sensing electrode for the detection of 4-NP in real applications.
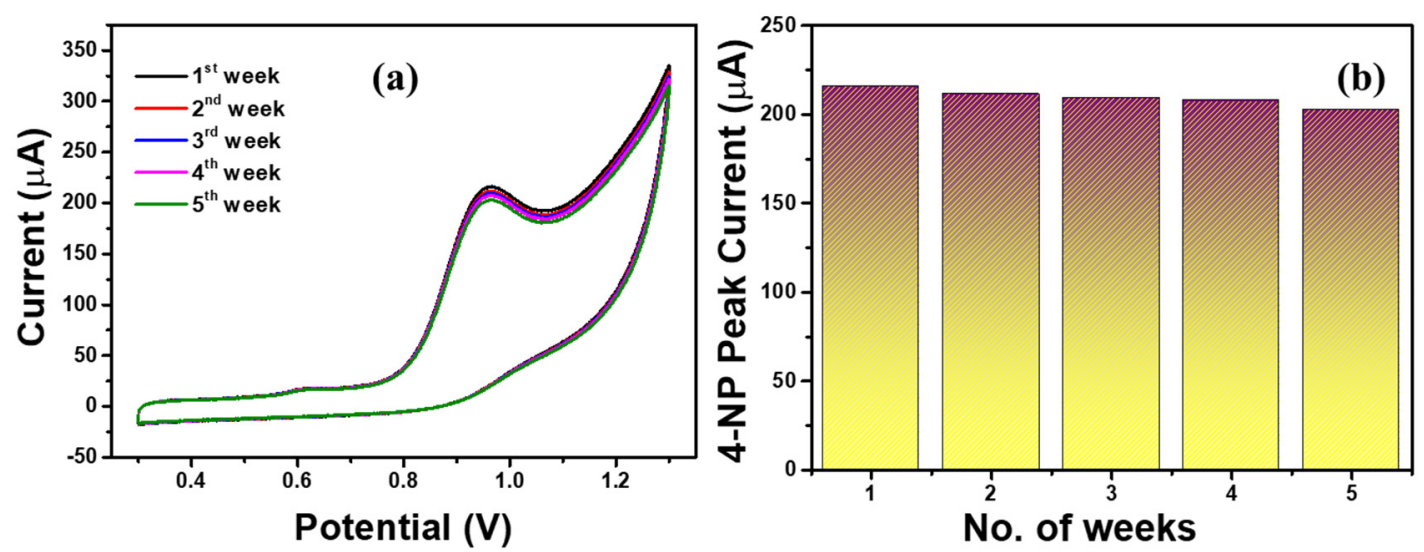

Figure 11. (a) Stability responses of Ag-ZnO/GCE (b) Ag-ZnO/GCE electrode response current in 500 $\mu \mathrm{M} 4-\mathrm{NP}$ containing $0.1 \mathrm{M}$ PBS ( $\mathrm{pH}=7$ ) solution. (Accumulation time: 2 min, catalyst loading volume: $10 \mu \mathrm{L}$ ).

In addition, the interference study was analyzed to understand the selectivity of the $\mathrm{Ag}-\mathrm{ZnO} / \mathrm{GCE}$ electrode with 4-NP containing inorganic/organic interference chemicals as shown in Figure 12. From Figure 12, it can be seen that the noticeable change in the 4-NP oxidation peak current, which confirmed the poor selectivity of the Ag-ZnO/GCE electrode towards 4-NP with interference chemicals; however, the Ag-ZnO/GCE electrode showed high sensitivity towards 4-NP in the neutral medium. In addition, the sensitivity of $\mathrm{Ag}-\mathrm{ZnO} / \mathrm{GCE}$ electrode was compared to previously reported sensor electrodes in Table 1 [40-43].

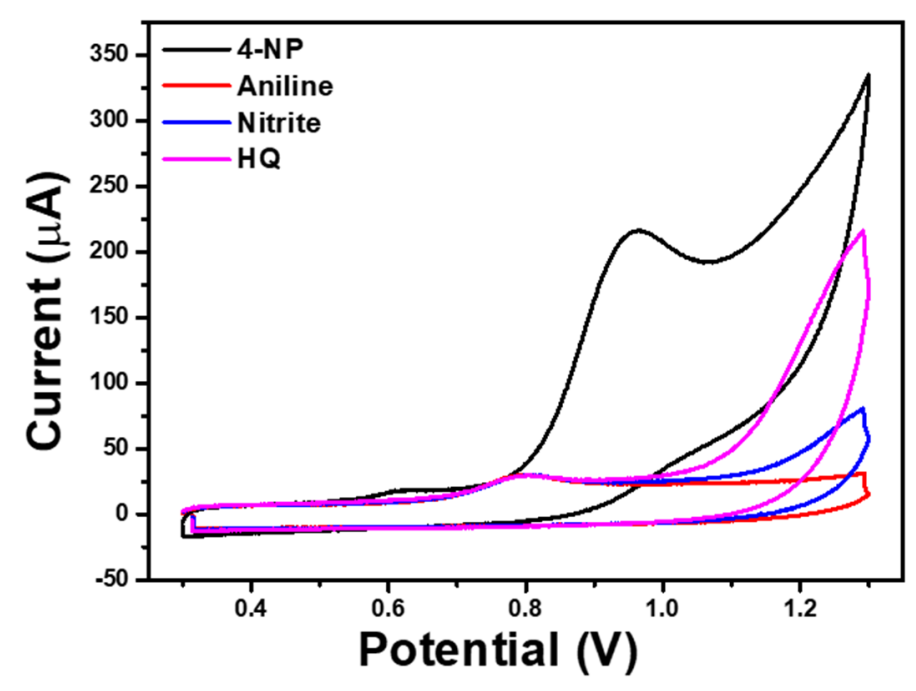

Figure 12. $\mathrm{CV}$ responses of Ag-ZnO/GCE in $500 \mu \mathrm{M}$ 4-NP containing with $500 \mu \mathrm{M}$ aniline, nitrite, hydroquinone in $0.1 \mathrm{M}$ PBS ( $\mathrm{pH}=7$ ) solution. (Accumulation time: $2 \mathrm{~min}$, catalyst loading volume: $10 \mu \mathrm{L})$. 
Table 1. Sensitivity comparison of the Ag-ZnO/GCE electrode on 4-NP.

\begin{tabular}{cccccc}
\hline Electrode & Method & $\begin{array}{c}\text { Detection } \\
\text { Potential }(\mathbf{V})\end{array}$ & $\begin{array}{c}\text { Linear Range } \\
(\mu \mathbf{M})\end{array}$ & $\begin{array}{c}\text { LOD } \\
(\mu \mathbf{M})\end{array}$ & Ref. \\
\hline $\mathrm{AuNP} / \mathrm{RGO}$ & $\mathrm{DPV}$ & -0.6 & $0.05-2.0$ & 0.01 & 40 \\
\hline $\mathrm{Ag} \mathrm{NWs}-\mathrm{PANI}$ & $\mathrm{DPV}$ & -0.77 & $0.6-32$ & 0.052 & 41 \\
\hline $\mathrm{GCE} / \mathrm{Fe}_{3} \mathrm{O}_{4}-\mathrm{Pt}$ & $\mathrm{DPV}$ & -0.73 & $0.1-1.5$ & 0.048 & 42 \\
\hline $\mathrm{Ag} / \mathrm{RGO}$ & $\mathrm{SWV}$ & -0.5 & $10-101$ & 0.0012 & 43 \\
\hline $\begin{array}{c}\mathrm{\gamma}-\mathrm{Fe}_{2} \mathrm{O}_{3}-\mathrm{N}-\mathrm{rGO} \\
\mathrm{hydrogel}\end{array}$ & $\mathrm{DPV}$ & $-0.3,-0.6$ & $1-1000$ & 0.1 & 7 \\
\hline $\mathrm{Ag}-\mathrm{ZnO} / \mathrm{GCE}$ & $\mathrm{LSV}$ & 0.96 & $10-500$ & 3 & This work \\
\hline
\end{tabular}

\section{Experimental Details}

\subsection{Materials}

All chemicals such as silver nitrate, zinc nitratehexahydrate, hexamethylenetetramine, ammonium hydroxide, PBS, potassium ferrric cyanide and 4-nitrophenol were procured from Sigma-Aldrich and used as procured without further purification.

\subsection{Synthesis of Ag-Doped Zno Nanoflowers Decorated with Nanosheets}

Ag-doped $\mathrm{ZnO}$ nanoflowers were synthesized by the facile hydrothermal method. Typically, $0.1 \mathrm{M} \mathrm{Zn}\left(\mathrm{NO}_{3}\right)_{2} \cdot 6 \mathrm{H}_{2} \mathrm{O}(40 \mathrm{~mL})$ and $0.05 \mathrm{M} \mathrm{AgNO}_{3}(40 \mathrm{~mL})$ solutions were mixed and vigorously stirred for $30 \mathrm{~min}$, then an aqueous solution of $0.1 \mathrm{M}$ HMTA $(20 \mathrm{~mL})$ was added and stirred for $30 \mathrm{~min}$. A few drops of $\mathrm{NH}_{4} \mathrm{OH}$ were added to the resultant solution to maintain the $\mathrm{pH}=11$, then stirred again for $30 \mathrm{~min}$. Consequently, the obtained solution was then poured into a Teflon-lined stainless steel autoclave and maintained at $160{ }^{\circ} \mathrm{C}$ for $6 \mathrm{~h}$. After that, the autoclave was lowered to room-temperature, and finally whitish-yellow colored precipitate was filtered and washed with DI water and ethanol thoroughly, then dried at $60^{\circ} \mathrm{C}$ for $3 \mathrm{~h}$.

\subsection{Characterizations of Ag-Doped $\mathrm{ZnO}$ Nanoflowers Decorated with Nanosheets}

The synthesized Ag-doped $\mathrm{ZnO}$ nanoflowers decorated with nanosheets were examined using several techniques to understand the morphological, crystal, structural, compositional and surface properties. X-ray diffraction (PAN analytical Xpert Pro.) was employed to scrutinize the crystal properties, such as crystallinity, purity, phases, etc. The morphology, elemental composition and elemental mapping of the prepared Ag-doped $\mathrm{ZnO}$ nanoflowers decorated with nanosheets were obtained from field emission scanning electron microscopy (FESEM: JEOL-JSM-7600F) and transmission electron microscopy (TEM: JEOL-JEM-2100F) equipped with high-resolution TEM (HRTEM). To study the chemical compositions, the prepared nanoflowers were examined by Fourier transform infrared spectroscopy (FTIR: Spectrum-100), measured in the range of $450-4000 \mathrm{~cm}^{-1}$ at roomtemperature using the $\mathrm{KBr}$ dispersion and palletization process. The optical properties were investigated by UV-Visible spectroscopy (UV Vis.: Perkin Elmer-UV-VIS-Lambda 950) in the range of 250-800 $\mathrm{nm}$ at room-temperature. X-ray photoelectron spectroscopy (XPS; AXIS-NOVA CJ109, Kratos Inc., San Diego, CA, USA) was utilized to analyze the binding energies and the existence of various oxidation states in the Ag-doped $\mathrm{ZnO}$ nanoflowers decorated with nanosheets.

\subsection{Electrochemical Sensing Applications of Ag-Doped ZnO Nanoflowers Decorated with Nanosheets}

All electrochemical studies such as cyclic voltammetry (CV), Linear sweep voltammetry (LSV) and Impedance analysis (EIS) were analyzed by using Princeton-applied research potential Stat 3 (AMETEK, Berwyn, PA, USA). For 4-NP sensor, three electrode systems with $\mathrm{Ag}-\mathrm{ZnO}$ modified GCE, $\mathrm{Pt}$ and $\mathrm{Ag} / \mathrm{AgCl}$ electrodes were used as working, counter 
and reference electrode respectively. 4-NP detection was analyzed in $0.1 \mathrm{M} \mathrm{PBS}(\mathrm{pH}=7)$ solution form the potential range of 0.25 to $1.3 \mathrm{~V}$ at a scan rate of $100 \mathrm{mV} \mathrm{s}^{-1}$. In addition, electrochemical activity and conductivity of Ag-ZnO/GCE electrode was analyzed in $5 \mathrm{mM}$ ferri-ferro solution.

\section{Fabrication of a Ag-Doped ZnO Nanoflowers-Modified Electrode}

Ag-doped $\mathrm{ZnO}$ nanoflowers were modified by the drop casting method. Prior to drop casting, the GCE electrode was smoothly cleaned with $0.05 \mu \mathrm{m}$ alumina paste on a polishing cloth to obtain a shiny surface. Then the electrode was washed with ethanol and water thoroughly. The $10 \mathrm{mg}$ Ag-doped $\mathrm{ZnO}$ nanoflowers were dispersed with $1 \mathrm{~mL}$ of $\mathrm{DI}$ water through ultra-sonication. After that, $10 \mu \mathrm{L}$ dispersion was drop coated on the precleared GCE electrode and allowed to evaporate the water molecule at room temperature for $2 \mathrm{~h}$. Thus, $\mathrm{Ag}-\mathrm{ZnO} / \mathrm{GCE}$ was fabricated and stored at room temperature after the sensing experiment.

\section{Conclusions}

In summary, Ag-doped $\mathrm{ZnO}$ nanoflowers decorated with nanosheets were synthesized by facile hydrothermal process and characterized in detail using different characterization techniques. The detailed characterizations confirmed that the synthesized material was flower-shaped morphologies decorated with nanosheets and mainly exhibiting the wurtzite hexagonal phase of $\mathrm{ZnO}$. The synthesized $\mathrm{ZnO}$ nanoflowers were used as a functional material to fabricate a highly sensitive and selective eco-toxic 4-nitrophenol chemical sensor over glassy carbon electrode. The fabricated chemical sensor exhibited more highsensitivity and selectivity than the bare GCE electrode. An Ag-doped ZnO/GCE-based 4-nitrophenol chemical sensor exhibited a wide range of sensitivity from $10 \mu \mathrm{M}$ to $500 \mu \mathrm{M}$ with a good detection limit of $3 \mu \mathrm{M}(\mathrm{S} / \mathrm{N}=3)$. The observed results confirmed that the fabricated $\mathrm{Ag}-\mathrm{ZnO} / \mathrm{GCE}$ electrode could be the promising sensing electrode for various toxic chemicals.

Author Contributions: Conceptualization, A.U., M.S.A., H.A., A.A.I. and S.B.; methodology, A.U., M.S.A., H.A., A.A.I. and S.B.; software, A.U., M.S.A., H.A., and M.A.M.A.; formal analysis, A.U., M.S.A., H.A., A.A.I. and S.B.; investigation, A.U., M.S.A., H.A., A.A.I., M.A.M.A. and S.B.; writingoriginal draft preparation, A.U.; writing—review and editing, A.U., M.S.A., H.A., A.A.I., M.A.M.A. and S.B. All authors have read and agreed to the published version of the manuscript.

Funding: This research was funded by the Deputy for Research and Innovation-Ministry of Education, Kingdom of Saudi Arabia for the research through a grant (NU/IFC/INT/01/004) under the institutional funding committee at Najran University, Kingdom of Saudi Arabia.

Institutional Review Board Statement: Not applicable.

Informed Consent Statement: Not applicable.

Data Availability Statement: Data is contained within the article.

Acknowledgments: The authors would like to acknowledge the support of the Deputy for Research and Innovation-Ministry of Education, Kingdom of Saudi Arabia for the research through a grant (NU/IFC/INT/01/004) under the institutional funding committee at Najran University, Kingdom of Saudi Arabia.

Conflicts of Interest: The authors declare no conflict of interest.

Sample Availability: The samples are available from the authors.

\section{References}

1. Aditya, T.; Pal, A.; Pal, T. Nitroarene reduction: A trusted model reaction to test nanoparticle catalysts. Chem. Commun. 2015, 51, 9410-9431. [CrossRef]

2. Sangili, A.; Annalakshmi, M.; Chen, S.M.; Balasubramanian, P.; Sundrarajan, M. Synthesis of silver nanoparticles decorated on core-shell structured tannic acid-coated iron oxide nanospheres for excellent electrochemical detection and efficient catalytic reduction of hazardous 4-nitrophenol. Compos. Part B Eng. 2019, 162, 33-42. [CrossRef] 
3. Yin, H.; Zhou, Y.; Ai, S.; Liu, X.; Zhu, L.; Lu, L. Electrochemical oxidative determination of 4-nitrophenol based on a glassy carbon electrode modified with a hydroxyapatite nanopowder. Microchim. Acta 2010, 169, 87-92. [CrossRef]

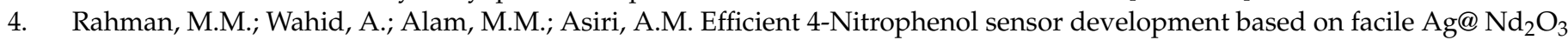
nanoparticles. Mater. Today Commun. 2018, 16, 307-313. [CrossRef]

5. Ramu, A.G.; Kumari, M.A.; Elshikh, M.S.; Alkhamis, H.H.; Alrefaei, A.F.; Choi, D. A facile and green synthesis of CuO/NiO nanoparticles and their removal activity of toxic nitro compounds in aqueous medium. Chemosphere 2021, 271, 129475. [CrossRef]

6. Gopi, S.; Giribabu, K.; Kathiresan, M. Porous organic polymer-derived carbon composite as a bimodal catalyst for oxygen evolution reaction and nitrophenol reduction. ACS Omega 2018, 3, 6251-6258. [CrossRef]

7. Ramu, A.G.; Salla, S.; Gopi, S.; Silambarasan, P.; Yang, D.J.; Song, M.J.; Ali, H.M.; Salem, M.Z.; Choi, D. Surface-tuned hierarchical $\mathrm{z}-\mathrm{Fe}_{2} \mathrm{O}_{3}-\mathrm{N}-\mathrm{rGO}$ nanohydrogel for efficient catalytic removal and electrochemical sensing of toxic nitro compounds. Chemosphere 2021, 268, 128853. [CrossRef]

8. Ramu, A.G.; Salla, S.; Chandrasekaran, S.; Silambarasan, P.; Gopi, S.; Seo, S.Y.; Yun, K.; Choi, D. A facile synthesis of metal ferrites and their catalytic removal of toxic nitro-organic pollutants. Environ. Pollut. 2021, 270, 116063. [CrossRef]

9. Gopi, S.; Perumal, S.; Al Olayan, E.M.; AlAmri, O.D.; Aloufi, A.S.; Kathiresan, M.; Yun, K. 2D Trimetal-organic framework derived metal carbon hybrid catalyst for urea electro-oxidation and 4-nitrophenol reduction. Chemosphere 2021, 267, 129243. [CrossRef]

10. Liu, B.; Wang, T.; Yin, C.; Wei, Z. Electrochemical analysis of p-nitrophenol in acidic or alkaline medium using silver nanoparticle decorated multi-walled carbon nanotubes. J. Mater. Sci. 2014, 49, 5398-5405. [CrossRef]

11. Liu, L.; Chen, R.; Liu, W.; Wu, J.; Gao, D. Catalytic reduction of 4-nitrophenol over Ni-Pd nanodimers supported on nitrogendoped reduced graphene oxide. J. Hazard. Mater. 2016, 320, 96-104. [CrossRef]

12. Xiao, N.; Liu, S.G.; Mo, S.; Li, N.; Ju, Y.J.; Ling, Y.; Li, N.B.; Luo, H.Q. Highly selective detection of p-nitrophenol using fluorescence assay based on boron, nitrogen co-doped carbon dots. Talanta 2018, 184, 184-192. [CrossRef]

13. Yu, H.; Merib, J.; Anderson, J.L. Crosslinked polymeric ionic liquids as solid-phase microextraction sorbent coatings for high performance liquid chromatography. J. Chromatogr. A 2016, 1438, 10-21. [CrossRef] [PubMed]

14. Padilla-Sánchez, J.A.; Plaza-Bolaños, P.; Romero-González, R.; Garrido-Frenich, A.; Vidal, J.L.M. Application of a quick, easy, cheap, effective, rugged and safe-based method for the simultaneous extraction of chlorophenols, alkylphenols, nitrophenols and cresols in agricultural soils, analyzed by using gas chromatography-triple quadrupole-mass spectrometry/mass spectrometry. J. Chromatogr. A 2010, 1217, 5724-5731. [PubMed]

15. Guo, X.; Wang, Z.; Zhou, S. The separation and determination of nitrophenol isomers by high-performance capillary zone electrophoresis. Talanta 2004, 64, 135-139. [CrossRef] [PubMed]

16. Barman, K.; Changmai, B.; Jasimuddin, S. Electrochemical detection of Para-nitrophenol using copper metal nanoparticles modified gold electrode. Electroanalysis 2017, 29, 2780-2787. [CrossRef]

17. da Silva, P.S.; Gasparini, B.C.; Magosso, H.A.; Spinelli, A. Gold nanoparticles hosted in a water-soluble silsesquioxane polymer applied as a catalytic material onto an electrochemical sensor for detection of nitrophenol isomers. J. Hazard. Mater. 2014, 273, 70-77. [CrossRef]

18. Theerthagiri, J.; Salla, S.; Senthil, R.A.; Nithyadharseni, P.; Madankumar, A.; Arunachalam, P.; Maiyalagan, T.; Kim, H.S. A review on $\mathrm{ZnO}$ nanostructured materials: Energy, environmental and biological applications. Nanotechnology 2019, 30, 392001. [CrossRef]

19. Arruebo, M.; Fernandez-Pacheco, R.; Irusta, S.; Arbiol, J.; Ibarra, M.R.; Santamaría, J. Sustained release of doxorubicin from zeolite-magnetite nanocomposites prepared by mechanical activation. Nanotechnology 2006, 17, 4057. [CrossRef]

20. Govindhan, M.; Lafleur, T.; Adhikari, B.R.; Chen, A. Electrochemical sensor based on carbon nanotubes for the simultaneous detection of phenolic pollutants. Electroanalysis 2015, 27, 902-909. [CrossRef]

21. Wei, Y.; Kong, L.T.; Yang, R.; Wang, L.; Liu, J.H.; Huang, X.J. Single-walled carbon nanotube/pyrenecyclodextrin nanohybrids for ultrahighly sensitive and selective detection of p-nitrophenol. Langmuir 2011, 27, 10295-10301. [CrossRef] [PubMed]

22. Zhang, X.F.; Liu, Z.G.; Shen, W.; Gurunathan, S. Silver nanoparticles: Synthesis, characterization, properties, applications, and therapeutic approaches. Int. J. Mol. Sci. 2016, 17, 1534. [CrossRef] [PubMed]

23. Umar, A.; Ibrahim, A.A.; Kumar, R.; Algadi, H.; Albargi, H.; Alsairi, M.A.; Alhmami, M.A.; Zeng, W.; Ahmed, F.; Akbar, S. $\mathrm{CdO}-\mathrm{ZnO}$ nanorices for enhanced and selective formaldehyde gas sensing applications. Environ. Res. 2021, 111377. [CrossRef] [PubMed]

24. Cheng, H.; Zhou, Z.; Qin, D.; Huang, W.; Feng, J.; Tang, T.; Hu, G.; Li, L. Electrochemical Sensor Based on Electrospun Three-Dimensional Carbon Nanofibers to Determine Trace Levels of Cu(II). Sci. Adv. Mater. 2020, 12, 693-700. [CrossRef]

25. Kumar, R.; Umar, A.; Kumar, R.; Chauhan, M.S.; Al-Hadeethi, Y. ZnO-SnO 2 nanocubes for fluorescence sensing and dye degradation applications. Ceram. Int. 2021, 47, 6201-6210. [CrossRef]

26. Hong, C.; Zhou, Q.; Lu, Z.; Umar, A.; Kumar, R.; Wei, Z.; Wu, X.; Xu, L.; Kim, S.H. Ag-doped ZnO nanoellipsoids based highly sensitive gas sensor. Mater. Express 2017, 7, 380-388. [CrossRef]

27. Al-Hadeethi, Y.; Umar, A.; Ibrahim, A.A.; Al-Heniti, S.H.; Kumar, R.; Baskoutas, S.; Raffah, B.M. Synthesis, characterization and acetone gas sensing applications of Ag-doped ZnO nanoneedles. Ceram. Int. 2017, 43, 6765-6770. [CrossRef]

28. Kumar, R.; Rana, D.; Umar, A.; Sharma, P.; Chauhan, S.; Chauhan, M.S. Ag-doped ZnO nanoellipsoids: Potential scaffold for photocatalytic and sensing applications. Talanta 2015, 137, 204-213. [CrossRef] [PubMed]

29. Kumar, M.; Chauhan, M.S.; Akhtar, M.S.; Umar, A. Effect of cerium ions in Ce-Doped ZnO nanostructures on their photocatalytic and picric acid chemical sensing. Ceram. Int. 2021, 47, 3089-3098. [CrossRef] 
30. Patil, S.S.; Mali, M.G.; Tamboli, M.S.; Patil, D.R.; Kulkarni, M.V.; Yoon, H.; Kim, H.; Al-Deyab, S.S.; Yoon, S.S.; Kolekar, S.S.; et al. Green approach for hierarchical nanostructured $\mathrm{Ag}-\mathrm{ZnO}$ and their photocatalytic performance under sunlight. Catal. Today 2016, 260, 126-134. [CrossRef]

31. Subhan, M.A.; Awal, M.R.; Ahmed, T.; Younus, M. Photocatalytic and antibacterial activities of Ag/ZnO nanocomposities fabricated by co-precipitation method. Acta Met. Sin. (Engl. Lett.) 2014, 27, 223-232. [CrossRef]

32. Shin, M.H.; Park, M.S.; Jung, S.H.; Boo, J.H.; Lee, N.E. Effect of doping elements on $\mathrm{ZnO}$ etching characteristics with $\mathrm{CH}_{4} / \mathrm{H}_{2} / \mathrm{Ar}$ plasma. Thin Solid Film. 2007, 515, 4950-4954. [CrossRef]

33. Thomas, M.A.; Sun, W.W.; Cui, J.B. Mechanism of Ag doping in ZnO nanowires by electrodeposition: Experimental and theoretical insights. J. Phys. Chem. C 2012, 116, 6383-6391. [CrossRef]

34. Wang, Z.G.; Zu, X.T.; Yang, S.Z.; Wang, L.M. Blue luminescence from carbon modified ZnO nanoparticles. J. Mater. Sci. 2006, 41, 3729-3733. [CrossRef]

35. Armelao, L.; Fabrizio, M.; Gialanella, S.; Zordan, F. Sol-gel synthesis and characterisation of ZnO-based nanosystems. Thin Solid Film. 2001, 394, 89-95. [CrossRef]

36. Arvinte, A.; Mahosenaho, M.; Pinteala, M.; Sesay, A.M.; Virtanen, V. Electrochemical oxidation of p-nitrophenol using graphenemodified electrodes, and a comparison to the performance of MWNT-based electrodes. Microchim. Acta 2011, 174, 337-343. [CrossRef]

37. Jiang, P.; Zhou, J.; Zhang, A.; Zhong, Y. Electrochemical degradation of p-nitrophenol with different processes. J. Environ. Sci. 2010, 22, 500-506. [CrossRef]

38. Perumal, S.; Moon, I.S. Sustainable NO removal and its sensitive monitoring at room temperature by electrogenerated Ni(I) electron mediator. Chemosphere 2021, 265, 129122. [CrossRef] [PubMed]

39. Silambarasan, P.; Moon, I.S. Real-time monitoring of chlorobenzene gas using an electrochemical gas sensor during mediated electrochemical degradation at room temperature. J. Electroanal. Chem. 2021, 894, 115372. [CrossRef]

40. Tang, Y.; Huang, R.; Liu, C.; Yang, S.; Lu, Z.; Luo, S. Electrochemical detection of 4-nitrophenol based on a glassy carbon electrode modified with a reduced graphene oxide/Au nanoparticle composite. Anal. Methods. 2013, 5, 5508-5514. [CrossRef]

41. Zhang, C.; Govindaraju, S.; Giribabu, K.; Huh, Y.S.; Yun, K. AgNWs-PANI nanocomposite based electrochemical sensor for detection of 4-nitrophenol. Sens. Actuators B Chem. 2017, 252, 616-623. [CrossRef]

42. Gerent, G.G.; Spinelli, A. Magnetite-platinum nanoparticles-modified glassy carbon electrode as electrochemical detector for nitrophenol isomers. J. Hazard. Mater. 2017, 330, 105-115. [CrossRef] [PubMed]

43. Ikhsan, N.I.; Rameshkumar, P.; Huang, N.M. Controlled synthesis of reduced graphene oxide supported silver nanoparticles for selective and sensitive electrochemical detection of 4-nitrophenol. Electrochim. Acta 2016, 192, 392-399. [CrossRef] 